\title{
Manejo de alopecia no paciente em tratamento oncológico
}

\section{Management of alopecia in oncologic treatment patients}

Karine Corcione Turke ${ }^{1}$, Jose Roberto Fraga Neto², Deli Brito de Oliveira ${ }^{3}$, Laissa Arruda Pinto ${ }^{4}$, Jean Schoueri ${ }^{1}$, Vanessa Salzano ${ }^{5}$ Dolores Gonzalez Fabra ${ }^{5}$

${ }^{1}$ Faculdade de Medicina do ABC (FMABC) - Santo André (SP), Brasil.

${ }^{2}$ Faculdade de Ciências Médicas da Santa Casa de São Paulo (FCMSCSP) - São Paulo (SP), Brasil.

${ }^{3}$ Curso em Processos Industriais, Instituto de Pesquisas Tecnológicas do Estado de São Paulo (IPT) - São Paulo (SP), Brasil.

${ }^{3}$ Curso em Processos Industriais, Instituto de Pesquisas Tecnologicas
${ }^{4}$ Departamento de Dermatologia, FMABC - Santo André (SP), Brasil.

${ }^{5}$ Ambulatório de Reabilitação Dermato-Cosmiátrica para Pacientes Oncológicos, FMABC - Santo André (SP), Brasil.

DOI: https://dx.doi.org/10.7322/abcshs.v44i3.1395

\section{RESUMO}

Introdução: O tratamento do câncer vem evoluindo a cada dia. Entretanto, efeitos adversos como alterações dermatológicas apresentam um grande impacto psicossocial ao paciente oncológico. Uma dessas alterações dermatológicas é a alopecia, caracterizada pela perda dos fios da sobrancelha e do couro cabeludo. Relato de caso: Foram referidos dois casos de pacientes em tratamento quimioterápico com alopecia parcial e total. Ambas receberam tratamento por meio do Minoxidil, de uso tópico, havendo repilação completa após três meses. Não foram observados efeitos adversos. Conclusão: A loção tópica de Minoxidil mostrou-se um método efetivo e seguro para tratar a alopecia parcial e total em pacientes que ainda estão em tratamento oncológico.

Palavras-chave: Alopecia; oncologia integrativa; dermatologia; tratamento farmacológico.

\section{ABSTRACT}

Introduction: The treatment of cancer has been evolving every day. However, adverse effects such as dermatological changes have a great psychosocial impact on cancer patients. One dermatological change is alopecia, characterized by loss of eyebrow and scalp strands. Case report: Two cases of patients undergoing chemotherapy with partial and total alopecia were reported. Both were treated with Minoxidil, which was topically used, with complete repilation after three months. No adverse effects observed. Conclusion: Minoxidil topical lotion has proven to be an effective and safe method to treat partial and total alopecia in patients still undergoing cancer treatment.

Keywords: alopecia; Integrative oncology; dermatology; drug therapy. 


\section{INTRODUÇÃO}

O câncer é uma doença que vem crescendo ano após ano, juntamente com a expectativa de vida populacional. Atualmente, é a segunda maior causa de morte, ficando atrás somente das doenças cardiovasculares ${ }^{1}$.

O surgimento de novos tratamentos oncológicos, complementares à quimioterapia e radioterapia, como terapia alvo e imunoterapia, por exemplo, aumentaram a expectativa e qualidade de vida dos pacientes oncológicos. Entretanto, assim como os tratamentos mais convencionais, há diversos efeitos adversos dermatológicos ${ }^{2,3}$.

Nas alterações dermatológicas encontram-se as capilares, que variam entre os tratamentos antineoplásicos. Essas mudanças incluem a alopecia, mas não se limitam a ela: mudanças de textura; tricomegalia e despigmentação capilar ${ }^{3}$.

Apesar dessas alterações não apresentarem riscos à saúde do paciente oncológico, há um grande impacto psicossocial e na autoestima, causando ansiedade e afetando a qualidade de vida relacionada à saúde ${ }^{4}$.

No caso de mulheres com câncer de mama, por exemplo, a alopecia é considerada, pelas pacientes, um efeito adverso traumático e estressante, podendo inclusive levar a uma recusa do tratamento. Há também relatos que a alopecia é uma experiência mais difícil que uma mastectomia radical, por exemplo ${ }^{4,5}$.

Dessa forma, é de extrema importância fornecer aos pacientes oncológicos o acesso à reabilitação dermato-cosmiátrica, de forma a reduzir a recusa e/ou o abandono do tratamento antineoplásico ${ }^{6}$.

Atualmente, existem alguns recursos para o manejo e tratamento dessas afecções. Por exemplo, o uso de resfriadores do couro cabeludo, como toucas. Por meio da redução do metabolismo das células do couro cabeludo, ou redução da perfusão sanguínea (com diminuição da concentração da quimioterapia no couro cabeludo), há menos queda de cabelo 7 .

Estudos recentes associaram o uso das toucas e métodos resfriadores com o aumento da incidência de metástases no couro cabeludo. O resfriamento pode fornecer um ambiente protetor para as micrometástases ${ }^{8,9}$.

O minoxidil vem sendo um forte aliado para esse efeito, pois é um ativo vasodilatador e atua aumentando a fase anágena de crescimento dos cabelos, contribuindo para o aumento da densidade capilar. As formulações tópicas disponíveis no mercado contendo minoxidil apresentam concentrações na faixa de $2 \%$ a $5 \%$ de ativo ${ }^{10}$.

O minoxidil é um fármaco vasodilatador que inicialmente foi utilizado para tratamento de hipertensão, porém, foi descoberto que um dos efeitos colaterais era o aumento de pelos pelo corpo. Seu mecanismo de ação tópica ainda não está claro, mas atua aumentando a fase anágena, contribuindo para o aumento da densidade capilar ${ }^{11}$.
Nesse relato, mostraremos a evolução da repilificação do couro cabeludo em uma paciente com alopecia total e uma com alopecia parcial, ambas tratadas com Minoxidil.

\section{RELATO DE CASO}

\section{Caso 1}

Paciente feminina, de 61 anos de idade, que foi diagnosticada com câncer de mama em outubro de 2016. Foi realizada mastectomia com linfadenectomia, seguida de quimioterapia - doxorrubicina, ciclofosfamida e taxol e, posteriormente, radioterapia, com 7 sessões. A paciente evoluiu com alopecia total, caracterizada pela perda da totalidade dos fios de cabelo no couro cabeludo e das sobrancelhas.

Durante o tratamento quimioterápico, foi utilizado Minoxidil a 5\% solução tópica, aplicada 2 vezes ao dia no couro cabeludo com realização de massagem suave pela paciente após a aplicação. Apesar da paciente ter realizado dermopigmentação de sobrancelhas, também foi orientada a usar Minoxidil nelas, sendo observada repilação significativa.

Observamos uma repilação de 100\% do couro cabeludo após 3 meses de uso do Minoxidil (Figura 1).

\section{Caso 2}

Paciente feminina, de 74 anos de idade, que foi diagnosticada com câncer de cólon em 2010, quando realizou quimioterapia durante 6 meses. Em 2016, após recidiva da lesão, iniciou cetuximabe, evoluindo com alopecia parcial importante, ou seja, apesar de perda significativa dos fios de cabelo do couro cabeludo, ainda ficaram mantidos parcialmente fios no couro cabeludo e nas sobrancelhas.

Durante o tratamento quimioterápico, foi utilizado Minoxidil a 5\% solução tópica, aplicada 2 vezes ao dia no couro cabeludo com realização de massagem suave pela paciente após a aplicação. Observamos uma repilificação de $100 \%$ do couro cabeludo após 3 meses de uso do Minoxidil (Figura 2)

\section{DISCUSSÃO}

A alopecia desencadeada pelo tratamento quimioterápico ou por terapias alvo é um efeito colateral comum que geralmente se inicia 1-3 semanas após o início do tratamento oncológico, sendo agravada com os ciclos subsequentes desse tratamento. Felizmente, esse quadro tem resolução espontânea após 3-6 meses do término do tratamento ${ }^{9}$.

Estima-se que essa condição atinja $65 \%$ dos pacientes em tratamento quimioterápico ou com terapia alvo. $47 \%$ das pacientes mulheres consideram a perda dos cabelos o mais traumático efeito colateral do tratamento, fazendo com que $8 \%$ delas cheguem 
a desistir do tratamento quimioterápico ou da terapia alvo por medo desse evento ${ }^{7}$.

O uso tópico do Minoxidil a $2 \%$ demonstrou ser útil por acelerar a recuperação da alopecia desencadeada por quimioterápicos e terapias alvo em paciente com câncer de mama que recebiam quimioterapia adjuvante e paciente com câncer ginecológico que receberam ciclofosfamida, doxorrubicina e cisplatina ${ }^{9}$.

Mesmo que o Minoxidil venha sendo usado há mais de 30 anos para estimular o crescimento dos cabelos em pacientes com alopecia androgenética, seu mecanismo de ação não está totalmente elucidado. Sabe-se que o Minoxidil encurta a fase telógena e acelera a entrada dos folículos pilosos em repouso na fase anágena. Além disso, o Minoxidil aumenta o tamanho dos folículos pilosos ${ }^{10}$.
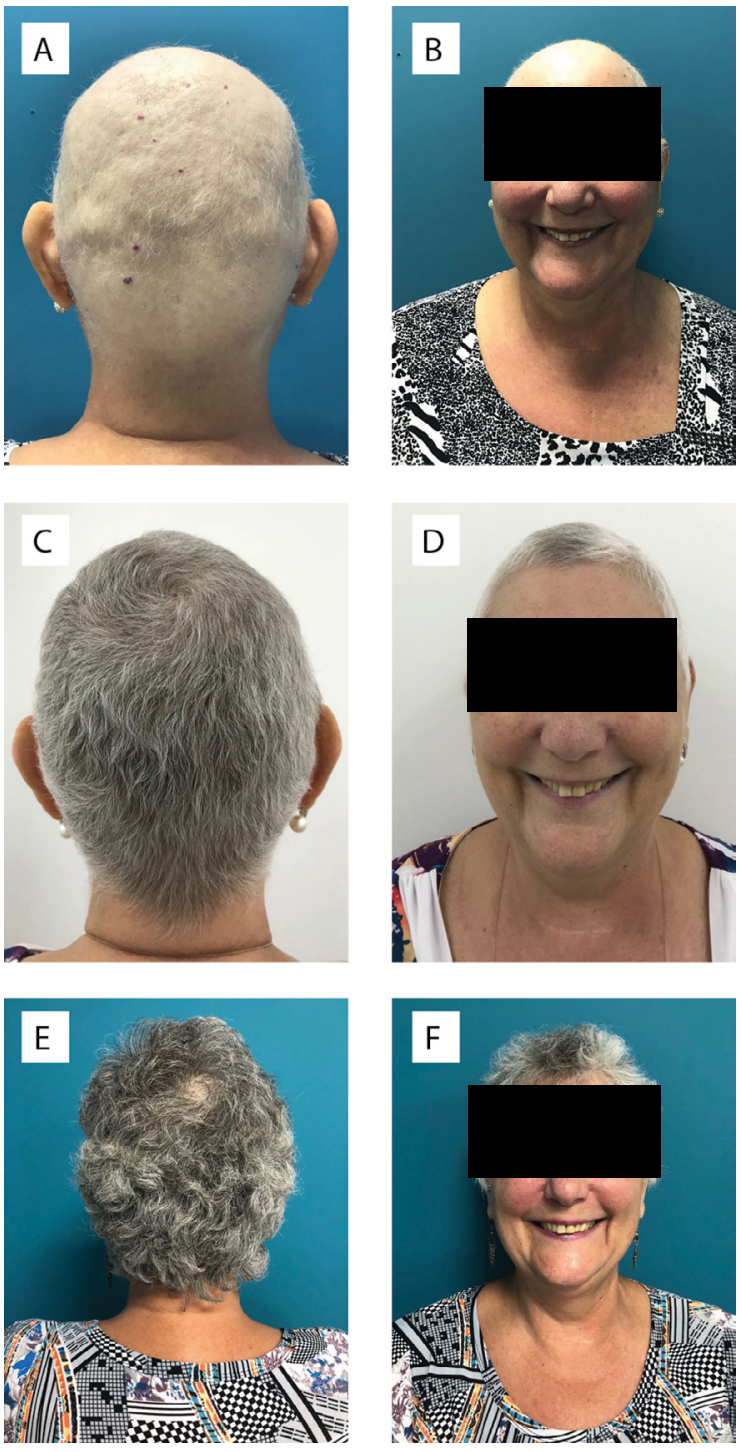

Figura 1: Evolução da paciente ao longo do tempo de uso do Minoxidil. A e B: prévio ao uso de Minoxidil; C e D: 45 dias de uso de Minoxidil; E e F: 3 meses de uso de Minoxidil.
O uso oral do Minoxidil causa diminuição da pressão sanguínea por relaxar a musculatura lisa dos vasos sanguíneos através da ação de seu metabólito sulfatado - Minoxidil sulfato - que age abrindo os canais de ATP-potássio. Existem evidências de que essa seja a ação do Minoxidil de uso tópico para crescimento dos cabelos, porém tem sido difícil demonstrar a expressão desses canais ATP-potássio no folículo piloso ${ }^{12}$.

Outra forma de ação seria sua atuação nas células mesenquimais da papila dérmica por ação indireta do Minoxidil, por vasodilatação e consequente aumento do aporte sanguíneo e até mesmo pela irritação local por ele desencadeada ${ }^{13}$.

Um estudo comparou o uso de Minoxidil nas concentrações de 2 e $5 \%$ e demonstrou que o grupo de pacientes que usaram
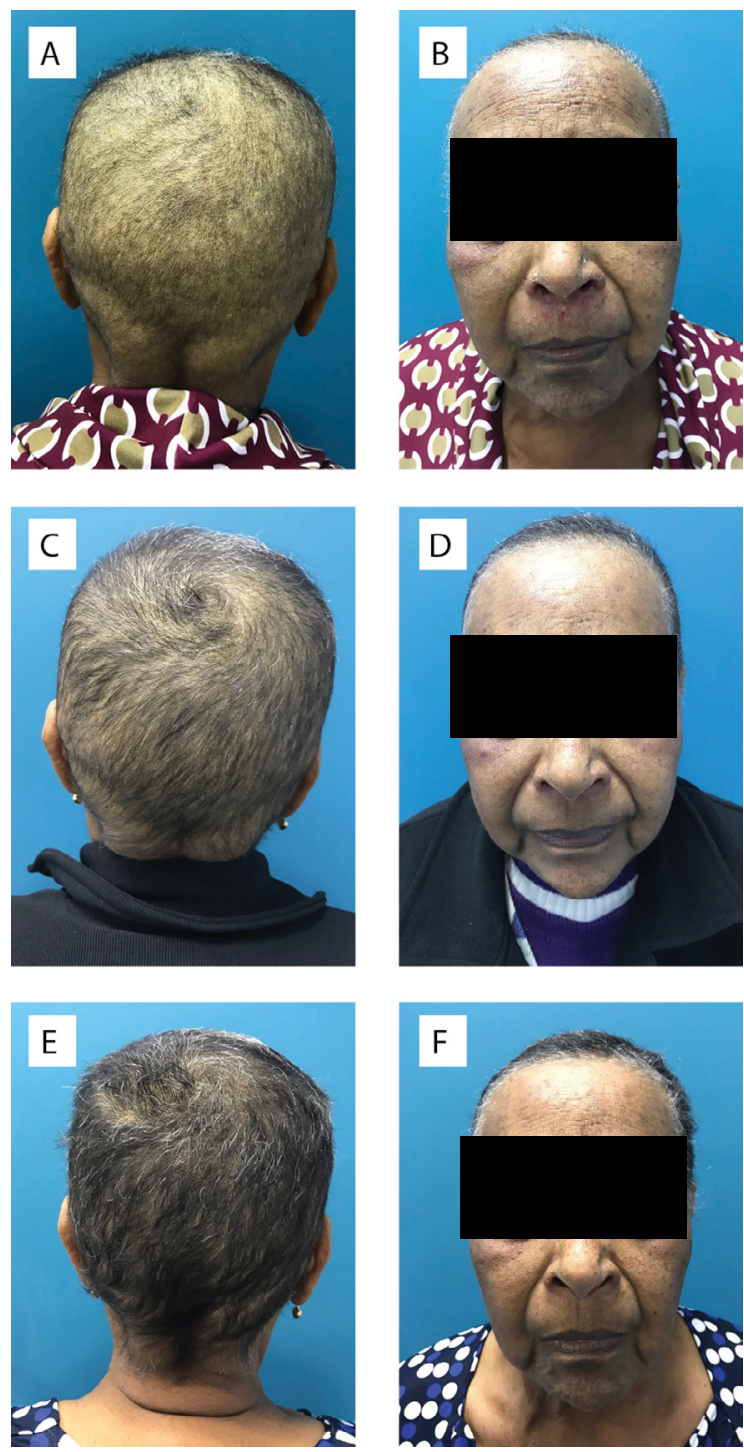

Figura 2: Evolução da paciente ao longo do tempo de uso do Minoxidil. A e B: prévio ao uso de Minoxidil; C e D: 45 dias de uso de Minoxidil; E e F: 3 meses de uso de Minoxidil. 
Minoxidil na concentração de 5\% apresentou resposta mais estatisticamente eficaz em 48 semanas que o grupo que usou a $2 \%$, porém ambos ajudaram a melhorar a percepção psicossocial das pacientes ${ }^{14}$.

Todavia, existem relatos de que o Minoxidil de uso tópico pode causar irritações locais e reações tipo dermatite de contato no couro cabeludo. Muitas vezes, esse quadro está mais relacionado ao propilenoglicol, que não é um principio ativo, mas que está frequentemente presente nas formulações galênicas ${ }^{15}$.

Não foi encontrado nenhum caso de alergia com o uso do Minoxidil na literatura. Observou-se que uso da loção de
Minoxidil a $2 \%$ nos pacientes em tratamento oncológico promoveu repilação efetiva, tanto nos casos de alopecia parcial, como nos casos de alopecia total, além de espessamento dos fios de cabelo do couro cabeludo e sobrancelha e redução da perda dos fios.

Dessa forma, a despeito dos efeitos colaterais relatados, resultados promissores observados na prática diária no Ambulatório de Reabilitação Dermato-Cosmiátrica para pacientes oncológicos da Faculdade de Medicina do ABC levam à conclusão que o uso do Minoxidil é um método efetivo para combater de forma segura a alopecia parcial e total em pacientes que ainda estão em tratamento oncológico.

\section{REFERÊNCIAS}

1. Ferlay J, Soerjomataram I, Dikshit R, Eser S, Mathers C, Rebelo M, et al. Cancer incidence and mortality worldwide: Sources, methods and major patterns in GLOBOCAN 2012. Int J Cancer. 2014;136:E359-86. https://doi.org/10.1002/ijc.29210

2. Fabra DG, Kayo AP, Leal ARO, Salzano V, Guglielmino F. Alterações dermatológicas em pacientes oncológicos- adultos e crianças. Arq Bras Ciên Saúde. 2009;34(2):87-93.

https://dx.doi.org/10.7322/abcs.v34i2.132

3. Cubero DIG, Abdalla BMZ, Schoueri J, Lopes FI, Turke KC, Guzman J, et al. Cutaneous side effects of molecularly targeted therapies for the treatment of solid tumors. Drugs in Context. 2018;7:212516.

https://dx.doi.org/10.7573/dic.212516

4. Choi EK, Kim IR, Chang O, Kang D, Nam SJ, Lee JE, et al. Impact of chemotherapy-induced alopecia distress on body image, psychosocial well-being and depression in breast cancer patients. Psychooncology. 2014;23(10):1103-10 https://dx.doi.org/10.1002/pon.3531

5. Freites-Martinez A, Shapiro J, Chan D, Fornier M, Modi S, Gajria D, et al. Endocrine therapy-induced alopecia in patients with breast cancer. JAMA Dermatol. 2018;154(6):670-5. https://dx.doi.org/10.1001/jamadermatol.2018.0454

6. Fabra DG, Salzano V, Guglielnino F, Kayo AP. Impacto do ambuatório de reabilitação dermatocosmiátrica na qualidade de vida dos pacientes oncológicos da Faculdade de Medicina do ABC. Arq Bras Ciên Saúde. 2010:35(2):89-94. https://dx.doi.org/10.7322/abcs.v35i2.92

7. Trueb RM. Chemotherapy-induced alopecia. Semin Cutan Med Surg. 2009;28:11-14. https://dx.doi.org/10.1016/j.sder.2008.12.001
8. Grevelman EG, Breed WPM. Prevention of chemotherapy- induced hair loss by scalp cooling. Ann Oncol. 2005;16(3):352-8 https://dx.doi.org/10.1093/annonc/mdi088

9. Shin H, Jo SJ, Kim DH, Kwon O, Myung SK. Efficacy of interventions for prevention of chemotherapy-induced alopecia: a systematic reviwe and meta-analysis. Int J Cancer. 2015;136(5):E442-54. https://dx.doi.org/10.1002/ijc.29115

10. Barbareschi M. The use of minoxidil in the treatment of male and female androgenetic alopecia: a story of more than 30 year. $\mathrm{G}$ Ital Dermatol Venereol. 2018;153(1):102-6. https://dx.doi.org/10.23736/S0392-0488.17.05781-9

11. Rey MCW, Bonamigo RR. Tratamento de alopecia areata. Med Cutánea Iber Lat Am. 2006;34(2):49-52.

12. Messenger AG, Rundegren J. Minoxidil: mechanisms of action on hair growth. Br J Dermatol. 2004;150(2):186-94

13. Headington JT. Hair follicle biology and topical minoxidil: possible mechanisms of action. Dermatologica. 1987;175(2):19-22. https://dx.doi.org/10.1159/000248894

14. Lucky AW, Piacquadio DJ, Ditre CM, Dunlap F, Kantor I, Pandya $A G$, et al. A randomized, placebo-controlled trial of $5 \%$ and $2 \%$ topical minoxidil solutions in the treatment of female pattern hair loss. J Am Acad Dermatol. 2004;50(4):541-53. https://dx.doi.org/10.1016/j.jaad.2003.06.014

15. Rossi A, Cantisani C, Melis L, Iorio A, Scali E, Calvieri S. Minoxidil use in dermatology, side effects and recent patents. Recent Pat inflamm Allergy Drug Discov. 2012;6(2):130-6. https://dx.doi.org/10.2174/187221312800166859 